\title{
Effect of microencapsulation on Saccharomyces cerevisiae var. boulardii viability in the gastrointestinal tract and level of some blood biochemical factors in wistar rats
}

\author{
Hassan Ghorbani-Choboghlo ${ }^{1}$, Donya Nikaein ${ }^{2,3}$, Ali-Reza Khosravi ${ }^{2,3}$, Reza Rahmani ${ }^{4}$, Zohreh \\ Farahnejad $^{1^{*}}$ \\ ${ }^{1}$ Department of Medical Mycology, AJA University of Medical Sciences, Tehran, Iran \\ ${ }^{2}$ Mycology Research Center, Faculty of Veterinary Medicine, University of Tehran, Tehran, Iran \\ ${ }^{3}$ Department of Microbiology and Immunology, Faculty of Veterinary Medicine, University of Tehran, Tehran, \\ Iran \\ ${ }^{4}$ Department of Biochemistry, School of Medicine, Tehran University of Medical Sciences, Tehran, Iran
}

Received: December 2018, Accepted: February 2019

\begin{abstract}
Background and Objectives: Probiotics are live microorganisms that, when administered in an adequate amount, confer a health benefit on the host through the gut. Saccharomyces cerevisiae is a widespread yeast found in nature. This microorganism has been used as a probiotic agent in recent years. In this study, the effect of microencapsulation on survival rate of $S$. cerevisiae var. boulardii in the simulated gastrointestinal tract medium and the impact of microencapsulated $S$. cerevisiae var. boulardii on some serum biochemical factors in a rat model was evaluated.

Materials and Methods: 30 male wistar rats were divided into three groups (control, rats receiving microencapsulated $S$. cerevisiae var. boulardii, and rats receiving $S$. cerevisiae var. boulardii alone). The probiotic was gavaged at a dosage of $2 \mathrm{gr} /$ $\mathrm{kg} \mathrm{BW}$ for 8 weeks. Blood was collected from rats at the end of the treatment period and biochemical factors were measured using Mancompany kits.

Results: The results showed a significant increase in viability of microencapsulated $S$. cerevisiae var. boulardii in comparison with free $S$. cerevisiae var. boulardii $(\mathrm{p}<0.05)$. Weight of rats in probiotic treated groups was significantly higher in comparison with the control group $(\mathrm{p}<0.05)$. Moreover, probiotic treatment reduced mean levels of triglycerides, cholesterol, free blood sugar and liver enzymes in rats.

Conclusion: Microencapsulation could increase the survival rate of yeast probiotics in the gastrointestinal tract; however, more studies are needed for better understanding of the exact effect of microencapsulation on probiotics' function.
\end{abstract}

Keywords: Saccharomyces cerevisiae var. boulardii; Probiotic; Microencapsulation; Blood biochemical factors

\footnotetext{
"Corresponding author: Zohreh Farahnejad, PhD, Department of Medical Mycology, AJA University of Medical Sciences, Tehran, Iran.

Tel: $+98-2188028350$

Fax: +98-2188028350

Email: zfarahnejad@yahoo.com
}

\section{INTRODUCTION}

Probiotics, as defined by WHO and FAO, are "live microorganisms which when administered in adequate amounts confer a health benefit on the host" (1). According to literature, the main benefit of pro- 
biotics is maintaining a healthy digestive tract and immune system (2-4). Today, there are many commercially available probiotics developed from different species of nonpathogenic bacteria specially Lactobacillus spp. and Bifidobacterium spp. There are also some nonbacterial probiotics including some yeast species (5).

The genus Saccharomyces is widespread yeast found in nature. This microorganism has been used as a probiotic agent in recent years (6). Saccharomyces boulardii, found in probiotic preparations, is now a strain of $S$. cerevisiae known as $S$. cerevisiae var. boulardii (7). Recent studies have shown that these two species are genetically identical (8). S. boulardii is known for its anti-inflammatory, immunomodulatory and microbiome regulation effects and it has been approved by food and drug administration (FDA) as generally regarded as safe (GRAS) (1). It has been shown that this yeast inhibits microbial translocation and secretion of inflammatory cytokines such as IL6 (2). This yeast can survive inside the gastrointestinal tract because it can endure high temperatures and low $\mathrm{pH}$ (3). It is used for treatment of diarrhea as well (4).

Although many studies focus on improving advantages of probiotics, it must be noted that the most important characteristic in a probiotic microorganism is to survive stomach passage and preserve its function and viability in the intestinal tract $(5,6)$. Thus, methods improving survival rate of probiotics have high priority. For this purpose, this study was designed to investigate the effect of microencapsulation on survival rate of $S$. boular$d i$ in simulated gastrointestinal tract condition and the impact of microencapsulated $S$. boulardi on some blood biochemical factors in the mentioned model $(7,8)$.

\section{MATERIALS AND METHODS}

Animal model. 30 male wistar rats weighing between 100-110 gr were used in this study. Rats were prepared from the Department of Laboratory Animals, Faculty of Veterinary Medicine, University of Tehran, Tehran, Iran. Rats were kept at intermittent lightening conditions at $20^{\circ} \mathrm{C}$. Standard pellet food and water was provided ad libitum. Rats divided into three groups each containing 10 rats: rats receiving microencapsulated $S$. boulardi, control group and rats receiving uncapsulated $S$. boulardii. Probiotic was gavaged to rats at the dosage of $2 \mathrm{gr} / \mathrm{kg}$ body weight for 8 weeks and Control group received normal saline (8).

Yeast strain. $S$. boulardii (ATCC 74068) was obtained from Mycology Research Center, Faculty of Veterinary Medicine, University of Tehran, Tehran, Iran. It was cultured on sabouraud's dextrose broth (Merck, Germany) supplemented with chloramphenicol $(0.05 \%)$ (SC) and shaker incubated at $28^{\circ} \mathrm{C}, 180$ rpm for $48 \mathrm{hrs}$. After incubation, cells were centrifuged at $800 \times \mathrm{g}$ for $10 \mathrm{~min}$ and washed three times with sterile phosphate buffered saline (PBS) before use.

Microencapsulation. Extrusion technique was used for microencapsulation of $S$. boulardii as described previously (9). In brief, cells were suspended in $2 \%$ sodium alginate solution. Then, this mixture was injected to $0.1 \mathrm{M}$ calcium chloride solution using a sterile insulin syringe. Formed droplets were isolated from suspension with centrifugation.

Viability of microencapsulated/ un-encapsulated $S$. boulardii. A gastrointestinal simulation environment (6) was used to evaluate the viability of microencapsulated/un-encapsulated $S$. boulardii. Cells were incubated in simulated medium with $\mathrm{pH}$ adjusted to 2 and 8 for $120 \mathrm{~min}$ at $28^{\circ} \mathrm{C}$. Samples were collected from suspension at 0, 30, 60 and $120 \mathrm{~min}$ after incubation. Total count was done by preparing 10 fold serial dilutions in peptone water culturing samples on SC agar at $28^{\circ} \mathrm{C}$ for $72 \mathrm{hrs}$. Viability was determined by determining the number of yeast cells grown on SC to Control (time 0 ).

Determining blood biochemical factors. Blood was collected from rats at days 0 and after probiotic treatment. Biochemical factors including free blood sugar, cholesterol, triglycerides, and liver enzymes (SGOT and SGPT) were measured using Man company (name of company, city, country??) kits according to the manufacturer's instructions and autoanalyser.

Statistical analysis. Data were analyzed using SPSS version 21. Analysis of variance (ANOVA) was used to compare the results and $p$ value of less than 0.05 considered significant. 


\section{RESULTS}

Size and shape of beads. Surface morphology and shape of the 50 randomly selected calcium alginate beads were determined using light microscopy at magnification of $400 \times$. The shape of the beads was generally spherical, sometimes elliptical with a mean diameter of 50-90 $\mu \mathrm{m}$. Internal appearance of coated beads at $400 \times$ showed that yeasts were distributed randomly in the alginate matrix (Fig. 1). The loss during encapsulation was very low due to gentle methods used.

Cell tolerance to gastrointestinal conditions and determination of total viable counts. Survival rate of encapsulated yeast cells in simulated gastric $\mathrm{pH}$ and intestinal bile salt solutions is shown in Table 1. No significant reduction in viable count was observed in free as well as encapsulated cells in distilled water $(\mathrm{pH}$ 6.5) after incubation for $2 \mathrm{hrs}$ (control) $(p>0.05)$. However variations in counts of free (un-encapsulated) and microencapsulated S. cerevisiae var boulardii during incubation in simulated gastric juices (SGJ) and simulated intestinal juices (SIJ) were significant $(\mathrm{p}<0.05)$. Although, results demonstrated that there were significant reductions in the number of free yeasts after immediate exposure to pHs 2 and 8, during 120 min incubation in simulated gastric and intestinal condition there was a significant increase in survival rate of yeast cells in the microcapsules compared to free cells ( $\mathrm{p}<0.05)$.

Viability of $S$. boulardii was determined in semi gastrointestinal conditions in acidic and basic $\mathrm{pH}$ in different times. The results showed a significant in-

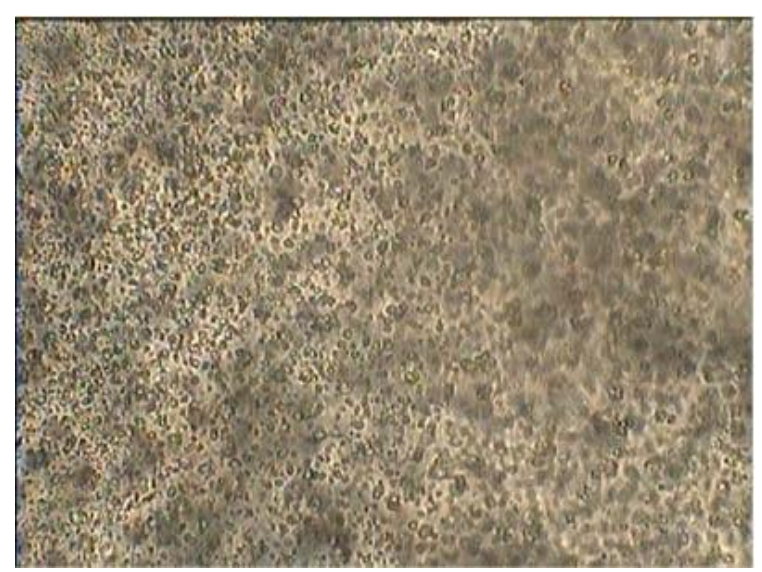

Fig. 1. Cross-section and internal appearance of yeast coated beads ( $\times 400$ magnification) crease in viability of microencapsulated $S$. boulardii in comparison with un-encapsulated $S$. boulardii ( $\mathrm{p}<0.05$ ) (Table 1). Reduction of $S$. boulardii count in basic media was lower than acidic condition. However this reduction was not significant $(\mathrm{p}>0.05)$.

Animals' weight gain. Rats were weighted at the end of experiment. Weight of rats in probiotic treated groups was significantly higher in comparison with control group $(\mathrm{p}<0.05)$ (Table 2). Liver weight was more in probiotic treated groups however this weight gain was not statistically significant $(\mathrm{p}>0.05)$.

Measurement of blood biochemical factors. Table 3 demonstrates the mean amount of certain biochemical factors in blood of different rats. Probiotic treatment reduced mean level of TGs, cholesterol, free blood sugar and liver enzymes in studied rats. These reductions were not significant between microencapsulated/un-encapsulated probiotic treated rats $(p>0.05)$ whilst in comparison with control group significant reduction was seen $(p<0.05)$.

\section{DISCUSSION}

Saccharomyces boulardii has been known as a nonpathogenic yeast with bio-therapeutic effects (17). The number of viable yeasts in the intestinal tract is critical to achieve yeasts efficacy, yet many of yeast cells do not survive the gastrointestinal tract (18). To maintain viability and function of probiotic, microencapsulation would be an appropriate method.

In the present study, effect of alkaline and acidic environment on viability of microencapsulated S. boulardii was investigated. According to the literature, these conditions are important in metabolic functions of microorganisms (19). In our study, alkaline $\mathrm{pH}(8)$ showed better results than acidic $\mathrm{pH}$. However this was not significant in microencapsulated $S$. boulardii. Duongthingoc et al. (2013) revealed that alkaline $\mathrm{pH}$ would restrict rapid proliferation of $S$. boulardii but maintain its CFU count (19). We demonstrated a reduction in all experiments $120 \mathrm{~min}$ utes after staying in an acidic and alkaline condition. The greatest reduction amount was for un-encapsulated $S$. boulardii in alkaline media, which shows lack of proliferation in this group. However, microencapsulation could improve yeast survival rate in different pH. Arslan et al. (2015) investigated the ef- 
Table 1. Viability of microencapsulated and unencapsulated S. boulardii in semi gastrointestinal condition in different times.

\begin{tabular}{llllllll}
\hline Media condition & Time (min) & $\mathbf{0}$ & $\mathbf{3 0}$ & $\mathbf{6 0}$ & $\mathbf{9 0}$ & $\mathbf{1 2 0}$ & Reduction amount \\
\hline Acidic & uncapsulated & 10 & 8 & 6 & 5 & 2.4 & 7.6 \\
Alkaline & microencapsulated & 10 & 10 & 8 & 7.3 & 6.1 & 3.9 \\
& uncapsulated & 10 & 7 & 5 & 4 & 2 & 8 \\
& microencapsulated & 10 & 9 & 7 & 6 & 6.4 & 3.6 \\
\hline
\end{tabular}

Table 2. Mean total weight and liver weight of rats studied after 8 weeks of treatment with microencapsulated/ un encapsulated probiotic

\begin{tabular}{lll}
\hline Group & Mean weight (gr) & Lier weight (mean) \\
\hline Control & 146.8 & 2.7 \\
Unencapsulated treated & 176 & 3.4 \\
Microencapsulated treated & 184.6 & 3.9 \\
\hline
\end{tabular}

Table 3. mean of studied biochemical factors' level in blood of different groups

\begin{tabular}{llll}
\hline Biochemical factor & Control & Microencapsulated & Un encapsulated \\
\hline Trigelycerides (mg/dl) & 76.3 & 62.3 & 58.2 \\
Cholesterol (mg/dl) & 62.7 & 57.3 & 51.2 \\
Free Blood Sugar (mg/dl) & 146 & 132 & 118 \\
SGPT/ALT (U/L) & 94 & 82 & 78 \\
SGOT/AST (U/L) & 162 & 151 & 143.2 \\
\hline
\end{tabular}

ficacy of different materials including gelatin, whey protein concentrate, modified starch, maltodextrin, pea protein isolate and gum arabic for microencapsulation of $S$. boulardii. Gum Arabic followed by gelatin and pea protein had the best survival rates at different $\mathrm{pH}$ levels (10). In our study, microencapsulation increased survival rate of yeast probiotic, however with increasing the exposure time, the survival rate decreased; which is compatible with other studies $(10,20)$.

Rats in the probiotic treated group had significantly more weight gain than the non-treated. Probiotics inhibit overgrowth of pathogens, modify gut microbiota and increase pancreatic digestive enzymes. These functions affect absorption of nutritional substances from intestines (21-23). More weight gain in $S$. boulardii treated rat would be as a result of this phenomenon. The role of yeast probiotics in protecting the liver is well known (24). S. cerevisiae var boulardii could improve liver function in rats treated by dimethyl nitrosamine (DMN) significantly (24).
In another study, a fermented substance from $S$. cerevisiae could protect rat liver from carbon tetrachloride $\left(\mathrm{CCL}_{4}\right)$ (25). In the current study, liver weight none significantly increased in probiotic treated rats in comparison with control groups and the amount of liver enzymes had decreased in probiotic treated rats. This is in concordance with other studies. We did not find significant differences in liver function between microencapsulated/un-encapsulated $S$. boulardii. Studies have shown that probiotic consumption would control hyperglycemia (26). This is similar to our findings in which rats fed with $S$. boulardii diet had lower levels of FBS. In addition, the amount of triglycerides and cholesterol in plasma had decreased as well which has been shown by other studies too $(27,28)$.

\section{CONCLUSION}

In conclusion, microencapsulation could increase 
survival rate of $S$. boulardii at different $\mathrm{pH}$ levels. Although microencapsulation could improve the effect of yeast probiotics on blood biochemical factors, we couldn't find significant differences in tested factors. Thus, more studies are needed to evaluate the effect of microencapsulation on $S$. boulardii functions in vivo.

\section{ACKNOWLEDGEMENTS}

The authors gratefully acknowledge the support provided by the research council of AJA University of Medical Sciences. Authors would also like to thank Mr. Asad Balal for his technical support in laboratory tests.

\section{REFERENCES}

1. Hill C, Guarner F, Reid G, Gibson GR, Merenstein DJ, Pot B, et al. Expert consensus document: the international scientific association for probiotics and prebiotics consensus statement on the scope and appropriate use of the term probiotic. Nat Rev Gastroenterol Hepatol 2014;11:506-514.

2. Ritchie ML, Romanuk TN. A meta-analysis of probiotic efficacy for gastrointestinal diseases.PLoS One 2012;7(4):e34938.

3. Bernaola Aponte G, Bada Mancilla CA, Carreazo NY, Rojas Galarza RA. Probiotics for treating persistent diarrhoea in children. Cochrane Database Syst Rev 2010;(11):CD007401.

4. AlFaleh K, Anabrees J. Probiotics for prevention of necrotizing enterocolitis in preterm infants. Evid Based Child Health 2014;9:584-671.

5. Tomičić ZM, Čolović RR, Čabarkapa IS, Vukmirović ĐM, Đuragić OM, Tomičić RM. Beneficial properties of probiotic yeast Saccharomyces boulardii. Food Feed Res 2016;43:103-110.

6. Kara I, Yıldırım F, Özgen Ö, Erganiş S, Aydoğdu M, Dizbay M, et al. Saccharomyces cerevisiae fungemia after probiotic treatment in an intensive care unit patient. J Mycol Med 2018;28:218-221.

7. Edwards-Ingram L, Gitsham P, Burton N, Warhurst G, Clarke I, Hoyle D, et al. Genotypic and physiological characterization of Saccharomyces boulardii, the probiotic strain of Saccharomyces cerevisiae. Appl Environ Microbiol 2007;73:2458-2467.

8. Profir A-G, Buruiana C-T, Vizireanu C. Effects of $S$. cerevisiae var. boulardii in gastrointestinal disorders.
J Agroaliment Proc Technol 2015;21:148-155.

9. Htwe K, Yee KS, Tin M, Vandenplas Y. Effect of Saccharomyces boulardii in the treatment of acute watery diarrhea in Myanmar children: a randomized controlled study. Am J Trop Med Hyg 2008;78:214-216.

10. Arslan S, Erbas M, Tontul I, Topuz A. Microencapsulation of probiotic Saccharomyces cerevisiae var. boulardii with different wall materials by spray drying. LWT Food Sci Technol 2015;63:685-690.

11. Martins FS, Vieira AT, Elian SD, Arantes RM, Tiago FC, Sousa LP, et al. Inhibition of tissue inflammation and bacterial translocation as one of the protective mechanisms of Saccharomyces boulardii against Salmonella infection in mice. Microbes Infect 2013;15:270279.

12. Graff S, Chaumeil J-C, Boy P, Lai-Kuen R, Charrueau C. Influence of $\mathrm{pH}$ conditions on the viability of Saccharomyces boulardii yeast. J Gen Appl Microbiol 2008; 54:221-227.

13. Ehrhardt S, Guo N, Hinz R, Schoppen S, May J, Reiser $\mathrm{M}$, et al. Saccharomyces boulardii to prevent antibiotic-associated diarrhea: a randomized, double-masked, placebo-controlled trial. Open Forum Infect Dis 2016;3(1):ofw011.

14. Dinleyici E, Kara A, Dalgic N, Kurugol Z, Arica V, Metin O, et al. Saccharomyces boulardii CNCM I-745 reduces the duration of diarrhoea, length of emergency care and hospital stay in children with acute diarrhoea. Benef Microbes 2015;6:415-421.

15. Van der Aa Kühle A, Skovgaard K, Jespersen L. In vitro screening of probiotic properties of Saccharomyces cerevisiae var. boulardii and food-borne Saccharomyces cerevisiae strains. Int J Food Microbiol 2005;101:29-39.

16. Ghorbani-Choboghlo H, Zahraei-Salehi T, Ashrafi-Helan J, Yahyaraeyat R, Pourjafar H, Nikaein D, et al. Microencapsulation of Saccharomyces cerevisiae and its evaluation to protect in simulated gastric conditions. Iran J Microbiol 2015;7:338-342.

17. Pozzoni P, Riva A, Bellatorre AG, Amigoni M, Redaelli E, Ronchetti A, et al. Saccharomyces boulardii for the prevention of antibiotic-associated diarrhea in adult hospitalized patients: a single-center, randomized, double-blind, placebo-controlled trial. Am J Gastroenterol 2012;107:922-931.

18. Klein SM, Elmer GW, McFarland LV, Surawicz CM, Levy RH. Recovery and elimination of the biotherapeutic agent, Saccharomyces boulardii, in healthy human volunteers. Pharm Res 1993;10:1615-1619.

19. Duongthingoc D, George P, Katopo L, Gorczyca E, Kasapis S. Effect of whey protein agglomeration on spray dried microcapsules containing Saccharomyces boulardii. Food Chem 2013;141:1782-1788.

20. Hébrard G, Hoffart V, Beyssac E, Cardot J-M, Alric M, 
Subirade M. Coated whey protein/alginate microparticles as oral controlled delivery systems for probiotic yeast. J Microencapsul 2010;27:292-302.

21. Buts J-P, Bernasconi P, Van Craynest M-P, Maldague $\mathrm{P}$, De Meyer R. Response of human and rat small intestinal mucosa to oral administration of Saccharomyces boulardii. Pediatr Res 1986;20:192-196.

22. Costalos C, Skouteri V, Gounaris A, Sevastiadou S, Triandafilidou A, Ekonomidou C, et al. Enteral feeding of premature infants with Saccharomyces boulardii. Early Hum Dev 2003;74:89-96.

23. Matur E, Ergul E, Akyazi I, Eraslan E, Cirakli Z. The effects of Saccharomyces cerevisiae extract on the weight of some organs, liver, and pancreatic digestive enzyme activity in breeder hens fed diets contaminated with aflatoxins. Poult Sci 2010;89:2213-2220.

24. Zoheir KM, Amara AA. The role of the yeast as probi- otic in protection against liver injury. Afr J Biotechnol 2012;11:16824-30.

25. Lai J-T, Hsieh W-T, Fang H-L, Lin W-C. The protective effects of a fermented substance from Saccharomyces cerevisiae on carbon tetrachloride-induced liver damage in rats. Clin Nutr 2009;28:338-345.

26. Ruan Y, Sun J, He J, Chen F, Chen R, Chen H. Effect of probiotics on glycemic control: a systematic review and meta-analysis of randomized, controlled trials. PLoS One 2015;10(7):e0132121.

27. Shareef A, Al-Dabbagh A. Effect of probiotic (Saccharomyces cerevisiae) on performance of broiler. Iraqi $J$ Vet Sci 2009;23:23-29.

28. Ooi L-G, Liong M-T. Cholesterol-lowering effects of probiotics and prebiotics: a review of in vivo and in vitro findings. Int J Mol Sci 2010;11:2499-2522. 